Ourania Papaioannou, Theodoros Karampitsakos, Matthaios Katsaras, Fotios Sampsonas, Argyrios Tzouvelekis

Department of Respiratory Medicine, University Hospital of Patras, University of Patras, Greece

\title{
Clinical improvement in Job syndrome following administration of co-trimoxazole, omalizumah and inhaled tobramycin
}

\begin{abstract}
Established treatment regimens for the autosomal dominant hyperimmunoglobulin $\mathrm{E}$ syndrome, denominated Job syndrome, are lacking. Thus, Job syndrome still exerts a dramatic impact on patients' quality of life. Our aim was to present safety and effectiveness of a regimen including co-trimoxazole, omalizumab and inhaled tobramycin in Job syndrome. A 26-year-old woman diagnosed with Job syndrome since infancy through sequencing revealing G342D mutation in STAT3 gene was initiated in the above mentioned treatment regimen; she was followed for 6 months, and to date, none recurrent pulmonary or skin infection was noticed. Furthermore, a considerable improvement in skin lesions was observed. A combination of anti-IgE and longitudinal use of inhaled antibiotics seems well-founded in Job syndrome.
\end{abstract}

Key words: Job syndrome, recurrent infections, omalizumab, inhaled antibiotics

\section{Introduction}

A 26-year-old woman, never smoker, presented to our department with cough, yellow sputum and localized left chest pain. Chest radiograph revealed consolidation in the left lower lobe and a fluid-filled cavity in the middle lobe. She denied the presence of fever, night sweats or weight loss. She had a medical history of Job syndrome diagnosed during infancy based on compatible genetic (G342D mutation in STAT3 gene) and clinical (recurrent lower respiratory and skin infections in need of repeated hospitalizations with several courses of empirical antimicrobial agents) findings.

\section{Material and methods}

\section{Physical examination findings}

Physical examination revealed the following vital signs: blood pressure of 110/70 mm Hg; heart rate, 85 beats/min; temperature, $36,8^{\circ} \mathrm{C}$ and oxygen saturation, $97 \%$ on room air. Lung auscultation revealed crackles mainly on left side while heart and abdomen examination results were unremarkable. There were no palpable lymph nodes. Clubbing was present, as well as extensive skin lesions on the upper limbs and hyperextensibility of finger joints.

\section{Diagnostic studies}

The complete blood and metabolic panel revealed elevated white blood cells (WBC) $(16,1 \mathrm{~K} / \mu \mathrm{L})$ with neutrophilic predominance on admission (62\%) and eosinophilic predominance on discharge $(40 \%)$ and elevated c-reactive protein $(22,33 \mathrm{mg} / \mathrm{dL})$. Urinalysis and electrocardiogram were normal. Chest computed tomography (CT) showed cystic bronchiectatic lesions in the right upper lobe combined with a fluid-filled cavity within the middle lobe and consolidation in the left lower lobe (Figure 1). The patient underwent conventional bronchoscopy with the presence of purulent bronchial secretions bilaterally and hemorrhagic mucosa in the middle lobe. Culture of washing for common pathogens was

Address for correspondence: Argyrios Tzouvelekis, Associate Professor of Respiratory Medicine, Head Department of Respiratory Medicine, University of Patras, Greece; e-mail: atzouvelekis@upatras.gr

DOI: 10.5603/ARM.a2021.0079 | Received: 24.07.2021 | Copyright (C 2021 PTChP | ISSN 2451-4934 | e-ISSN 2543-6031

This article is available in open access under Creative Common Attribution-Non-Commercial-No Derivatives 4.0 International (CC BY-NC-ND 4.0) license, allowing to download articles and share them with others as long as they credit the authors and the publisher, but without permission to change them in any way or use them commercially. 

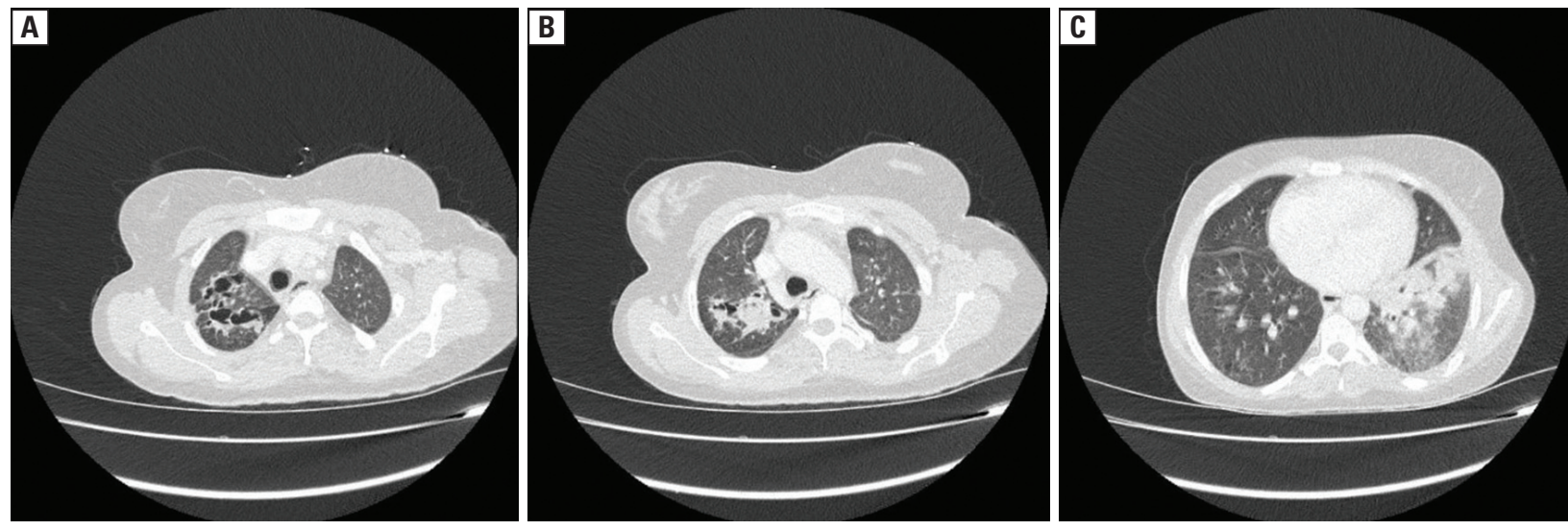

Figure 1. Chest computed tomography showed cystic bronchiectasis in the right upper lobe (A), a fluid-filled cavity in the middle lobe (B) and consolidation in the left lower lobe (C)

positive for Methicillin-resistant Staphylococcus aureus (MRSA) infection and negative for mycobacterium infection. Cytological examination of washing was negative for malignancy. Pulmonary function tests showed bronchodilator reversibility (PRE: forced expiratory volume in 1 second $\left[\mathrm{FEV}_{1}\right]$ / forced vital capacity [FVC]: $83 \%, \mathrm{FEV}_{1}$ of $1.65 \mathrm{~L}$ [59\% of predicted] and FVC of $2 \mathrm{~L}$ [63\% of predicted] - POST: $\mathrm{FEV}_{1} / \mathrm{FVC}: 87 \% \mathrm{FEV}_{1}$ of $1.8 \mathrm{~L}$ [ $68 \%$ of predicted] and FVC of $2.06 \mathrm{~L}$ [66\% of predicted]). Skin prick test was positive for mixed fungus, Tilia tomentosa and Candida. Moxifloxacin and linezolid were administered for ten days based on antibiogram. An improvement on chest radiogram performed four weeks later was obvious. This was the sixth hospitalization for our patient within the previous year attributed to pneumonia following multiple hospitalizations since infancy due to recurrent lower respiratory or skin infections.

\section{Clinical course}

The patient was admitted with recurrent pneumonia in setting of diagnosed Job syndrome since infancy. Genetic confirmation of the disease had been conducted through sequencing revealing G342D mutation in STAT3 gene. Recent laboratory examination had revealed remarkably elevated serum IgE levels $>30000 \mathrm{IU} / \mathrm{mL}$ and eosinophilia (31\% WBC count: $9.27 \mathrm{~K} / \mu \mathrm{L}$ ). During her last hospitalization, the patient was treated with antimicrobial therapy based on antibiogram, as part of symptomatic management of recurrent respiratory infections observed throughout the clinical course of syndrome. Considering multiple hospitalizations and extensive post-infectious structural abnormalities of lung parenchyma, multidisciplinary approach yielded a therapeutic regimen including biological anti-IgE agent and longitudinal antibiotics on a chemoprophylactic basis as the optimal strategy. In particular, the patient was commenced on co-trimoxazole, omalizumab and inhaled tobramycin. Established evidence has shown that co-trimoxazole is a safe and effective substitution to penicillins and has anti-MRSA coverage [1]. Furthermore, omalizumab, a monoclonal anti-IgE, has been shown closely related to a decline in serum IgE with symptomatic improvement especially in atopic conditions [2-4]. Eventually, inhaled antibiotics have emerged as a new option for specific groups of patients, including patients with bronchiectasis, combining high drug concentrations directly to the site of infection and concomitantly minimizing systemic absorption and potential side effects. Co-trimoxazole was administered orally in prophylactic dosage three times a week, omalizumab in dosage adjusted by the patient's weight and baseline IgE once a month subcutaneously and inhaled tobramycin $300 \mathrm{mg}$ twice a day. The woman was followed for 6 months, with no recurrent respiratory or skin infections. Furthermore, a considerable improvement of skin lesions was observed (Figure 2). To the extent of our knowledge, this is the first time that combination of anti-IgE and longitudinal use of inhaled antibiotics is proposed in Job syndrome.

\section{Discussion}

Hyperimmunoglobulin E syndrome is a rare primary immunodeficiency disorder characterized by eczema, skin abscesses, recurrent staphylococcal infections of the skin and lungs, pneumatocele formation, candidiasis, eosinophilia, and elevated serum levels of IgE [5]. It was 

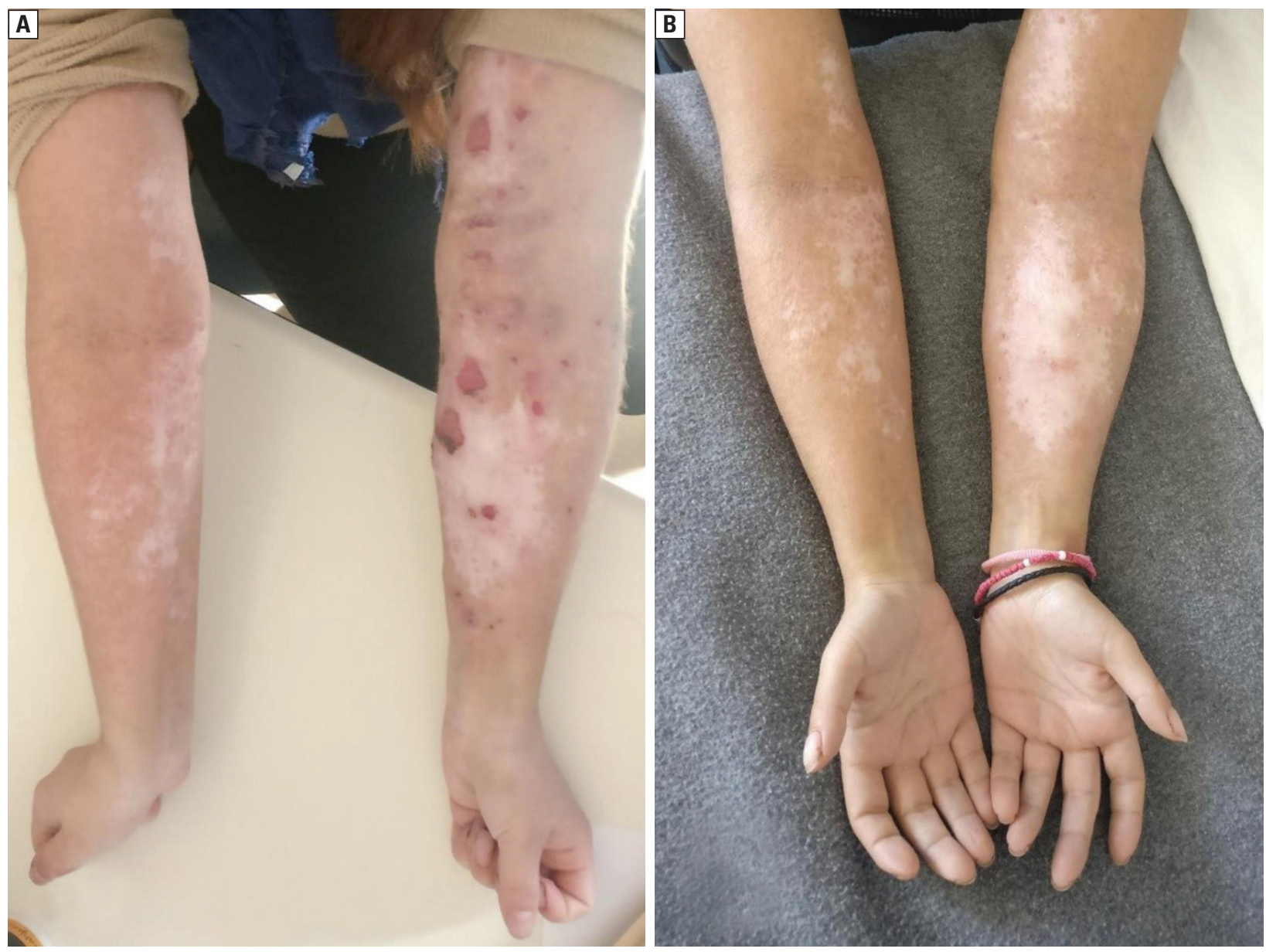

Figure 2. Improvement of skin lesions following administration of omalizumab for 6 months

first described as "Job syndrome" by David et al. in 1966 in two patients with eczema, recurrent pulmonary infections and cold lung abscesses and was later associated by Buckley et al. in 1972 with increased serum immunoglobulin E levels [6]. Job syndrome is classified as the autosomal dominant hyper-IgE syndrome, in which patients have abnormalities in different systems, including the immune system, connective tissue, skeletal and vascular structures [7]. It is attributed to a mutation in the STAT3 gene in more than two-thirds of cases (70\%) with the etiology of the rest cases remaining unclear [8]. Clinical signs of immunological features include recurrent skin and pulmonary bacterial or candidiasis infections. Staphylococcus aureus is the predominant pathogen resulting in follicular skin lesions or recurrent lung abscesses, bronchiectasis and pneumatoceles. Streptococcus pneumoniae and Haemophilus are observable less frequently, while Aspergillus and Pseudomonas are usual cause of chronic colonization of bronchiectasis. The main laboratory diagnostic feature is an increase in serum IgE levels of more than $2000 \mathrm{IU} / \mathrm{mL}$. Eosinophilia is observed in more than $90 \%$ of the patients while WBC count can be normal, elevated or reduced in number [9]. The established treatment is the long-term, sometimes consecutive, use of antibiotics by adapting the administration of antimicrobial agents to opportunistic infections occurring in affected patients, and in selected cases, applying surgical procedures during abscess development. In order to overcome purely symptomatic treatment applied for the moment, biological treatment with monoclonal antibodies or combination of biologics and antibiotics could be an optimal strategy. Further studies towards this direction are greatly anticipated.

\section{Conflict of interest}

None to declare.

\section{References:}

1. Hattori K, Hasui M, Masuda K, et al. Successful trimethoprim-sulfamethoxazole therapy in a patient with hyperimmu- 
noglobulin E syndrome. Acta Paediatr. 1993; 82(3): 324-326, doi: 10.1111/j.1651-2227.1993.tb12674.x, indexed in Pubmed: 8495097.

2. Casale TB. Anti-immunoglobulin E (omalizumab) therapy in seasonal allergic rhinitis. Am J Respir Crit Care Med. 2001; 164(8 Pt 2): S18-S21, doi: 10.1164/ajrccm.164.supplement 1.2103023, indexed in Pubmed: 11704613.

3. Milgrom H, Berger W, Nayak A, et al. Treatment of childhood asthma with anti-immunoglobulin E antibody (omalizumab). Pediatrics. 2001; 108(2): E36, doi: 10.1542/peds.108.2.e36, indexed in Pubmed: 11483846.

4. Alonso-Bello CD, Jiménez-Martínez MD, Vargas-Camaño ME, et al. Partial and transient clinical response to omalizumab in IL-21-induced low STAT3-phosphorylation on hyper-IgE syndrome. Case Reports Immunol. 2019; 2019: 6357256, doi: 10.1155/2019/6357256, indexed in Pubmed: $\underline{31355024}$.

5. Donabedian H, Alling DW, Gallin JI. Levamisole is inferior to placebo in the hyperimmunoglobulin E recurrent-infection (Job's) syndrome. N Engl J Med. 1982; 307(5): 290-292, doi: 10.1056/NEJM198207293070506, indexed in Pubmed: $\underline{6806658 .}$.

6. Grimbacher B, Holland SM, Gallin JI, et al. Hyper-IgE syndrome with recurrent infections--an autosomal dominant multisystem disorder. N Engl J Med. 1999; 340(9): 692-702, doi: 10.1056/ NEJM199903043400904, indexed in Pubmed: 10053178.

7. Gernez Y, Freeman AF, Holland SM, et al. Autosomal dominant hyper-IgE syndrome in the USIDNET registry. J Allergy Clin Immunol Pract. 2018; 6(3): 996-1001, doi: 10.1016/j. jaip.2017.06.041, indexed in Pubmed: 28939137.

8. Goel S, Sahu S, Minz RW, et al. STAT3-mediated transcriptional regulation of osteopontin in STAT3 loss-of-function related hyper IgE syndrome. Front Immunol. 2018; 9: 1080, doi: 10.3389/fimmu.2018.01080, indexed in Pubmed: 29868029.

9. Hashemi H, Mohebbi M, Mehravaran S, et al. Hyperimmunoglobulin E syndrome: Genetics, immunopathogenesis, clinical findings, and treatment modalities. J Res Med Sci. 2017 22: 53, doi: 10.4103/jrms.JRMS 1050 16, indexed in Pubmed 28567072 . 